\title{
Terapia nutricional en el soporte vital extracorpóreo
}

\author{
Nutritional therapy in extracorporeal life support \\ Terapia nutricional no suporte de vida extracorporal
}

\author{
Victoria Rebagliati ${ }^{*}$. \\ Recibido: 21 de noviembre de 2020. Aceptado para publicación: 19 de diciembre de 2020. \\ Publicado primero en línea: 19 de diciembre de 2020. \\ https://doi.org/10.35454/rncm.v4n3.237
}

\section{Resumen}

Los pacientes que requieren de soporte vital extracorpóreo se ubican entre los enfermos más críticos de la terapia intensiva. Actualmente se reconoce que la desnutrición proteica en los pacientes críticos puede prolongar los días en asistencia respiratoria mecánica (ARM) y la estadía en terapia intensiva, así como también aumentar la mortalidad y las complicaciones a largo plazo. Sin embargo, como la oxigenación extracorpórea es una técnica relativamente nueva, la evidencia en nutrición es muy escasa, por lo que surgen miedos al momento de alimentar artificialmente a estos pacientes. La presente revisión bibliográfica pretende responder los interrogantes sobre el abordaje nutricional adecuado basado en evidencia científica y la propia experiencia de un centro de referencia cardiovascular. La terapia nutricional debe llevarse a cabo en forma interdisciplinaria entre nutricionista, médico y enfermero con el fin de garantizar la colaboración de todos para alcanzar las metas nutricionales $y$, de este modo, prevenir la desnutrición.

Palabras clave: terapia médica nutricional, asistencia circulatoria mecánica, oxigenación extracorpórea, desnutrición.

\section{Abstract}

Patients that need extracorporeal life support are amongst the most critically ill in the intensive care unit (ICU). It is known that undernutrition in critical patients may reduce ventilator-free days, extend the length of stay, increase mortality and long-term complications. However, as extracorporeal oxygenation is a rather new tecnique, there is not enough evidence about nutrition, and that causes fear when these patients are artificially fed. The following review intends to answer doubts concerning nutrition therapy based on scientific evidence and the experience of a cardiovascular institute. Nutrition therapy must be carried out within interdisciplinary work between the nutritionist, doctor and nurse, so that with everyone's effort then nutritional targets may be accomplished and help to prevent undernutrition.

Keywords: Nutrition Medical Therapy; Mechanical Circulatory Assistance; Extracorporeal oxygenation; Undernutrition.

\section{Resumo}

Os pacientes que necessitam de suporte de vida extracorpóreo estão entre os pacientes mais críticos em terapia intensiva. Agora é reconhecido que a desnutrição protéica em pacientes críticos pode prolongar os dias de ventilação mecânica (ARM) e permanência em terapia intensiva, assim como aumentar a mortalidade e as complicações a longo prazo. Entretanto, como a oxigenação extracorpórea é uma técnica relativamente nova, as evidências na nutrição são muito escassas, de modo que os medos surgem quando se alimentam artificialmente esses pacientes. A presente revisão da literatura visa responder a perguntas sobre a abordagem nutricional apropriada com base em evidências científicas e na experiência de um centro de referência cardiovascular. A terapia nutricional deve ser realizada de forma interdisciplinar entre nutricionista, médico e enfermeiro, a fim de garantir a colaboração de todos para atingir os objetivos nutricionais e, assim, prevenir a desnutrição.

Palavras-chave: terapia de nutrição médica, assistência circulatória mecânica, oxigenação extracorporal, desnutrição.
Instituto Cardiovascular de Buenos Aires, Universidad Católica de la Plata. Grupo de Estudio de Paciente Crítico de la Asociación Argentina de Nutrición Enteral y Parenteral, Buenos Aires, Argentina.
*Correspondencia: Victoria Rebagliati. vrebagliati@icba.com.ar 


\section{INTRODUCCIÓN}

La oxigenación por membrana extracorpórea, más conocida como ECMO, es un dispositivo de asistencia circulatoria mecánica (ACM) que brinda soporte hemodinámico mediante la oxigenación y eliminación de dióxido de carbono $\left(\mathrm{CO}_{2}\right)$ en pacientes con insuficiencia pulmonar o cardíaca. Existen 2 técnicas distintas: la ECMO venovenosa (VV), que se utiliza en pacientes con insuficiencia pulmonar, y la venoarterial (VA), indicada para aquellos con insuficiencia cardíaca (Figura 1$)^{(1)}$.

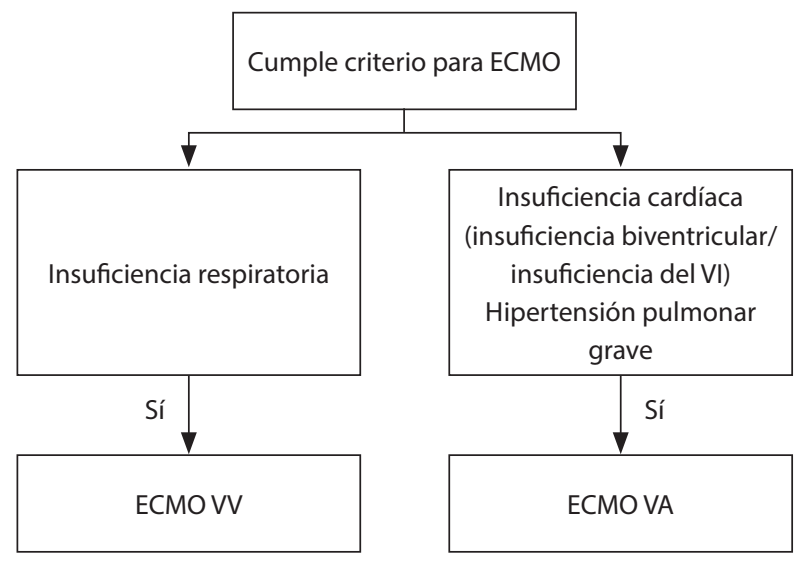

Figura 1. Algoritmo de elección para el tipo de soporte con ECMO.

La ECMO VA provee soporte cardíaco y respiratorio asegurando una adecuada perfusión sanguínea a órganos vitales en pacientes en choque cardiogénico. Está indicado en pacientes con patologías clínicas como infarto agudo de miocardio, miocarditis, intoxicación con fármacos cardiotóxicos y miocardiopatía dilatada en su etapa final; y patologías quirúrgicas como la poscardiotomía o choque cardiogénico postrasplante cardíaco, refractario al tratamiento convencional. Mayormente, se indica en pacientes con choque cardiogénico refractario y falla multiorgánica como último recurso, debido a esto es su elevada mortalidad. Frente a este panorama, la ECMO VA se utiliza como puente para la toma de decisiones entre la recuperación de la función cardíaca, el trasplante cardíaco, la ACM de largo plazo o el retiro del dispositivo en caso de futilidad ${ }^{(2)}$.

Los pacientes con ECMO se encuentran entre los más graves dentro de la población de enfermos críticos, dada la complejidad de su tratamiento. Suelen tener estancias más prolongadas en la unidad de cuidados intensivos (UCI), requieren mayor tiempo en rehabilitación y presentan un incremento en la morbimor- talidad $^{(3)}$. Sin embargo, no existen guías de nutrición específicas para pacientes adultos que requieran ECMO. En las guías publicadas en 2017 por la organización Extracorporeal Life Support Organization (ELSO), su única mención al manejo nutricional es que "como en todos los pacientes críticos, el soporte nutricional total en calorías y proteínas es esencial" ${ }^{(4)}$. Además, la evidencia científica sobre nutrición y ECMO es escasa: hasta la fecha, tan solo 7 estudios se han llevado a cabo desde 2004.

\section{VÍA DE ALIMENTACIÓN Y MOMENTO DE INICIO}

En cuanto a la vía de alimentación, aunque no existen estudios aleatorizados ni prospectivos al respecto, las guías de la European Society of Intensive Care Medicine (ESICM) 2017 mediante estudios observacionales sugieren que la alimentación enteral es factible en estos pacientes y recomiendan que la misma sea precoz ${ }^{(5)}$. De la misma manera, las guías de la European Society of Parenteral and Enteral Nutrition (ESPEN) publicadas en 2018 dictan que la alimentación enteral precoz debe realizarse en pacientes que reciben $\mathrm{ECMO}^{(6)}$. $\mathrm{Al}$ igual que para el resto de los pacientes críticos, se considera alimentación enteral precoz al inicio, dentro de las primeras 24 a 48 horas de su ingreso a la unidad de terapia intensiva (UTI). Por su parte, Stoppe sugiere iniciar la alimentación enteral lo antes posible luego de la colocación del dispositivo de ECMO y una vez controlado el choque y la estabilidad hemodinámica ${ }^{(3)}$.

Ohbe y colaboradores, en un estudio retrospectivo de 1769 pacientes que requirieron ECMO VA, encontraron que aquellos que recibieron nutrición enteral precoz ( $\mathrm{n}$ $=220$ ) tuvieron menor mortalidad tanto hospitalaria como a 28 días, que el grupo de pacientes que recibió nutrición enteral a partir del tercer día $(n=1549)$; y estos resultados fueron estadísticamente significativos ${ }^{(7)}$.

Desde nuestra experiencia, coincidimos en que la alimentación enteral precoz es factible en los pacientes con ECMO VA.

\section{COMPLICACIONES GASTROINTESTINALES}

El compromiso circulatorio y la inflamación, junto con el alto requerimiento de fármacos vasopresores y la anticoagulación en estos pacientes, hacen inherente el riesgo de alteración en la integridad gastrointestinal y en la perfusión esplácnica; por esta razón, muchos pacientes con ECMO presentan intolerancias gastrointesti- 
nales. Aunque no hay datos de su prevalencia, Ferrie y colaboradores reportaron intolerancias digestivas en un $38 \%$ de sus pacientes en los primeros 5 días $^{(8)}$; mientras que Lukas y colaboradores encontraron que solo el $55 \%$ de los pacientes en ECMO alcanzaron la meta calórica por complicaciones gastrointestinales ${ }^{(9)}$. Cabe destacar que, en el estudio llevado a cabo por Lukas, consideraban intolerancia digestiva a un volumen de residuo gástrico mayor de $200 \mathrm{~mL}$. Actualmente no se recomienda la medición de rutina del residuo gástrico como método para valorar la tolerancia digestiva ${ }^{(10)}$. Por su parte, en 2019 MacGowan y colaboradores analizaron la adecuación calórico-proteica de 203 pacientes con ECMO y hallaron que un tercio de los pacientes recibió un aporte de calorías y proteínas inadecuado, y que dicho déficit se asoció significativamente a la gravedad y falla multiorgánica de los pacientes, especialmente en los primeros 3 días. Uno de los principales motivos para el aporte insuficiente fue la gastroparesia (en $22,8 \%$ de los pacientes), pero el motivo principal fue las interrupciones en la alimentación por procedimientos médicos (en un 39,1\% de los casos) ${ }^{(11)}$.

Lo anterior lleva a pensar en estrategias para evitar el déficit nutricional, como puede ser la alimentación basada en el volumen a administrar en vez de indicar una velocidad de infusión fija, siempre y cuando el paciente presente una adecuada tolerancia digestiva.

Cabe considerar que muchos de los pacientes que requieran ECMO presentan insuficiencia cardíaca crónica (ICC) avanzada y se estima que un $33 \%$ manifiesta signos de caquexia cardíaca. Esto podría agravar la intolerancia digestiva a la alimentación dado el edema intestinal y la congestión hepática que suelen exhibir los pacientes con ICC avanzada ${ }^{(3)}$. Además, el deterioro nutricional previo sumado a la demanda metabólica de la ECMO y la dificultad para su alimentación por las complicaciones gastrointestinales aumentan la morbimortalidad en estos pacientes. Es de suma importancia valorar el estado nutricional de los pacientes con ECMO y trabajar para mantenerlo o recuperarlo ya que probablemente se sometan a un trasplante cardíaco. Hasta la fecha no existen herramientas de valoración nutricional validadas para esta población.

Teniendo en cuenta que la función del soporte extracorpóreo es mejorar la perfusión sanguínea, se puede suponer que dicha terapia mejoraría la integridad de la barrera intestinal. En $2015 \mathrm{Ni}$ y colaboradores llevaron a cabo un estudio en un modelo de porcinos con dificultad respiratoria y encontraron que en el grupo en tratamiento con ECMO la concentración de las proteínas en las uniones estrechas de la mucosa colónica y yeyunal se encontraba significativamente aumentada. Por lo anterior, concluyeron que la terapia de ECMO tendría un efecto protector sobre la mucosa intestinal ${ }^{(12)}$. Por su parte, en 2014 Zhao y colaboradores investigaron el efecto de la reanimación con ECMO sobre la isquemia intestinal en un modelo de conejos con choque hemorrágico prolongado. Evaluaron el grado de inflamación sistémica con la medición sérica del factor de necrosis tumoral alfa (TNF- $\alpha$ ) y lactato, y hallaron valores séricos significativamente menores en el grupo con ECMO. A su vez, midieron el daño intestinal (por medio del puntaje de Chiu) y encontraron que este fue más grave en el grupo control. También analizaron la expresión de proteínas de las uniones estrechas y fue mayor en el grupo con ECMO. De esta manera, demostraron que el soporte extracorpóreo redujo la inflamación sistémica y alivió el daño intestinal provocado por el choque hemorrágico prolongado. Sin embargo, las implicancias de estos hallazgos en humanos no han sido investigadas ${ }^{(13)}$.

\section{REQUERIMIENTOS NUTRICIONALES}

El efecto del soporte extracorpóreo sobre el gasto energético no ha sido suficientemente estudiado ${ }^{(14)}$. El estándar de oro para el cálculo de calorías en pacientes críticos es la calorimetría indirecta, que analiza la producción de $\mathrm{CO}_{2}$. Sin embargo, en pacientes en soporte extracorpóreo no es posible llevar a cabo dicha técnica, ya que el $\mathrm{CO}_{2}$ removido por la membrana extracorpórea no puede ser identificado por el calorímetro ${ }^{(3)}$. Esto se debe a que cuentan con 2 sitios de intercambio gaseoso: el pulmón y la membrana del circuito ECMO, por lo que deberían medirse por separado y luego combinarlos para insertar en la ecuación de Weir para el cálculo del gasto energético (GE). Para esto, Wollersheim y colaboradores elaboraron un protocolo para la medición del GE en pacientes con síndrome de dificultad respiratoria aguda (SDRA) bajo ECMO VV. Tomaron muestras de sangre antes y después de su paso por la membrana, realizaron un análisis de los gases en dichas muestras y los expresaron como valores absolutos de oxígeno $\left(\mathrm{O}_{2}\right)$ y $\mathrm{CO}_{2}$ por litro de sangre. Posteriormente, calcularon la diferencia entre el volumen del gas antes y después de la membrana y la multiplicaron por el flujo por minuto de la ECMO, expresando la tasa metabólica de la ECMO en $\mathrm{O}_{2} \mathrm{~mL} /$ min y $\mathrm{CO}_{2} \mathrm{~mL} / \mathrm{min}$. Sumaron estos valores con el volumen de $\mathrm{O}_{2}$ y $\mathrm{CO}_{2}$ pulmonar obtenidos por la calorimetría indirecta para luego estimar el GE por medio de la ecuación de Weir (Figura 2) ${ }^{(15)}$. 


\section{Fórmula de Weir}

Tasa metabólica

$(\mathrm{cal} / \mathrm{min})=3,94 \times \mathrm{VO}_{2}(\mathrm{~mL} / \mathrm{min})+1,11 \mathrm{VCO}_{2}(\mathrm{~mL} / \mathrm{min})$

Figura 2. Fórmula de Weir. $\mathrm{VCO}_{2}$ : tasa de producción de dióxido de carbono en mililitros por minuto; $\mathrm{VO}_{2}$ : consumo de oxígeno en mililitros por minuto.

Sería necesario validar dicho protocolo mediante calorimetría directa en un mayor número de pacientes y también en aquellos con asistencia circulatoria por ECMO VA. A su vez, mediante este protocolo compararon los resultados del GE obtenido en pacientes con SDRA sin ECMO frente a pacientes con SDRA y ECMO. Encontraron que el GE en aquellos con ECMO fue mayor, aunque sin significancia estadística, probablemente por el escaso volumen de pacientes estudiados; por lo que se necesitan mayores investigaciones para confirmar los datos obtenidos.

Otro hallazgo por el mismo grupo fue que al comparar el GE medido con su protocolo con las fórmulas predictivas (como Harris Bennedict), los resultados eran muy dispares y el $48 \%$ sobreestimaba el GE en 500 kcal. Sumado a esto, al comparar el GE con la fórmula simplificada de $25 \mathrm{kcal} / \mathrm{kg}$, que habitualmente se utiliza en ausencia de calorimetría indirecta, esta demostró subestimar significativamente el $\mathrm{GE}^{(15)}$.

Poco antes, De Waele describió otro método para calcular el GE, pero en un solo paciente con ECMO. En este caso, obtuvo un consumo de $19 \mathrm{kcal} / \mathrm{kg}$, llamativamente es un valor muy distinto a lo hallado por Wollersheim ${ }^{(16)}$.

En cuanto al requerimiento proteico, según Wollersheim, sería el mismo que el recomendado para todos los pacientes críticos por las guías ASPEN 2016; es decir, 1,2-2 g/ kg/día ${ }^{(10,14)}$. Sin embargo, recientemente Pelekhaty y colaboradores demostraron mediante el estudio de balance nitrogenado que los pacientes con ECMO VV requieren un aporte proteico mayor al recomendado para pacientes críticos. Para ello, llevaron a cabo un estudio con 29 pacientes, 17 de ellos eran obesos y 12 no obesos, que recibían un aporte proteico de $2 \mathrm{~g} / \mathrm{kg}$ de peso ideal y $2,1 \mathrm{~g} / \mathrm{kg} /$ día, respectivamente. Ambos grupos obtuvieron balance de nitrógeno negativo, $-2,2 \mathrm{~g} /$ día para los no obesos y -7,3 $\mathrm{g} /$ día para los obesos. Se demostró que los pacientes con soporte por circulación extracorpórea presentan un estado catabólico mayor y necesitarían un aporte de proteínas más elevado que el recomendado habitualmente para los enfermos críticos ${ }^{(17)}$.
Con respecto al aporte de lípidos, se debe tener en cuenta que los pacientes bajo tratamiento con ECMO cuentan con un sistema capilar artificial. La administración de emulsiones lipídicas en estos pacientes se ha asociado con un aumento en la coagulación y aglutinación dentro del circuito, incluso con dosis suficientes de anticoagulantes. Por tanto, aunque con poca evidencia (reporte de un caso), algunos autores no recomiendan la administración de emulsiones lipídicas en forma endovenosa en estos pacientes ${ }^{(14)}$. Sin embargo, dada la escasa información disponible, tampoco es posible realizar una recomendación basada en la evidencia sobre el uso apropiado de nutrición parenteral en pacientes que reciben ECMO.

Existe una nueva generación de membranas del oxigenador que podrían no tener este riesgo, aunque esto no ha sido estudiado aún. Teniendo en cuenta lo expuesto, se debe evaluar el riesgo-beneficio antes de indicar la nutrición parenteral en estos pacientes. Y en el caso de que la nutrición parenteral se considere un beneficio fundamentado por intolerancia a la nutrición enteral, se deben monitorizar de cerca los circuitos del oxigenador $^{(18)}$. En nuestra experiencia, la nutrición parenteral complementaria a la vía enteral es de gran ayuda para evitar el déficit calórico-proteico frente a las intolerancias digestivas.

\section{¿EXISTE PÉRDIDA DE NUTRIENTES?}

Aunque otros autores sugieren que podría haber pérdida de nutrientes en el circuito sanguíneo a través de la ECMO, solo un grupo de investigadores australianos abordó el interrogante. Estensen y colaboradores demostraron que el uso de ECMO se acompaña de una pérdida tanto de macro- como de micronutrientes. Para ello, llevaron a cabo un estudio cuyo objetivo era identificar si existe una reducción significativa en la concentración plasmática de macro- y micronutrientes en un modelo ex vivo de circuito con ECMO, al que administraron plasma humano entero durante 24 horas y tomaron muestras pre- y poscircuito. Encontraron reducciones significativas para algunos aminoácidos: alanina, arginina, cisteína, glutamina e isoleucina; vitaminas A y E; y glucosa; aunque el mecanismo para explicar tal pérdida requiere de mayor investigación, ya que no se pudo establecer una correlación con las propiedades químicas de los nutrientes como su polaridad, solubilidad o peso molecular. Sin embargo, tanto la reducción de glucosa como de arginina y glutamina podrían tener explicación: la disminución de glucosa podría deberse en parte al metabolismo celular; 
mientras que la menor concentración de arginina y glutamina se explicaría por su conversión a ornitina y glutamato, respectivamente ${ }^{(19)}$.

Se necesitan más investigaciones sobre las alteraciones metabólicas y la pérdida real de nutrientes por ECMO para poder compensar nutricionalmente a los pacientes y valorar el impacto sobre los resultados clínicos.

\section{CONCLUSIÓN}

Los pacientes sometidos a ECMO se encuentran entre los enfermos críticos de mayor gravedad, por lo que su nutrición es de suma importancia. Según las últimas recomendaciones, la nutrición enteral precoz es segura y debe iniciarse en las primeras 48 horas. Resulta recurrente la dificultad para alcanzar las metas nutricionales por intolerancias digestivas; en dichos casos se debe considerar el uso de nutrición parenteral complementaria. Cabe destacar que es importante contar con un equipo interdisciplinario de soporte nutricional para la correcta toma de decisiones frente a estos pacientes de difícil manejo. A pesar de esto, aún quedan interrogantes a resolver como los requerimientos nutricionales y la pérdida o no de nutrientes a través de la membrana; por lo que se necesitan más estudios aleatorizados para conocer si se está alimentando correctamente a nuestros pacientes con ECMO.

\section{Referencias bibliográficas}

1. Chauhan S, Subin S. Extracorporeal membrane oxygenationAn anesthesiologist's perspective-Part II: Clinical and technical consideration. Ann Card Anaesth. 2012;15(1):69-82. doi: 10.4103/0971-9784.91485

2. de Chambrun MC, Bréchot N, Combes A. Venoarterial extracorporeal membrane oxygenation in cardiogenic shock: indications, mode of operation, and current evidence. Curr Opin Crit Care. 2019;25(4):397-402. doi: 10.1097/ MCC.0000000000000627

3. Stoppe C, Nesterova E, Elke G. Nutritional support in patients with extracorporeal life support and ventricular assist devices. Curr Opin Crit Care. 2018;24(4):269-76. doi: 10.1097/ MCC.0000000000000512

4. Extracorporeal Life Support Organization (ELSO). General Guidelines for all ECLS Cases [Internet]; 2017 [consultado el 28 de junio de 2018]. Disponible en: https://www.elso. org/Portals/0/ELSO\%20Guidelines\%20General\%20All\%20 ECLS\%20Version\%201_4.pdf

5. Blaser AR, Starkopf J, Alhazzani W, Berger MM, Casaer MP, Deane AM, et al. Early enteral nutrition in critically ill patients: ESICM clinical practice guidelines. Intensive Care Med. 2017;43(3):380-98. doi: 10.1007/s00134-016-4665-0
6. Weimann A, Braga M, Carli F, Higashiguchi T, Hübner M, Klek S, et al. ESPEN guideline: Clinical nutrition in surgery. 2017;36(3):623-50. doi: 10.1016/j.clnu.2017.02.013

7. Ohbe H, Jo T, Yamana H, Matsui H, Fushimi K, Yasunaga H. Early enteral nutrition for cardiogenic or obstructive shock requiring venoarterial extracorporeal membrane oxygenation: a nationwide inpatient database study. Intensive Care Med. 2018;44(8):1258-65. doi: 10.1007/s00134-018-5319-1

8. Ferrie S, Herkes R, Forrest P. Nutrition support during extracorporeal membrane oxygenation (ECMO) in adults: a retrospective audit of 86 patients. Intensive Care Med. 2013;39(11):1989-94. doi: 10.1007/s00134-013-3053-2

9. Lukas G, Davies AR, Hilton AK, Pellegrino VA, Scheinkestel $\mathrm{CD}$, Ridley E. Nutritional support in adult patients receiving extracorporeal membrane oxygenation. Crit Care Resusc. 2010;12(4):230-4.

10. McClave SA, Taylor BE, Martindale RG, Warren MM, Johnson DR, Braunschweig C, et al. Guidelines for the Provision and Assessment of Nutrition Support Therapy in the Adult Critically ill Patient: Society of Critical Care Medicine (SCCM) and American Society for Parenteral and Enteral Nutrition (A.S.P.E.N.). JPEN J Parenter Enteral Nutr. 2016;40(2):159-211. doi: 10.1177/0148607115621863

11. MacGowan L, Smith E, Elliott-Hammond C, Sanderson B, Ong D, Daly K, et al. Adequacy of nutrition support during extracorporeal membrane oxygenation. Clin Nutr. 2019;38(1):324-31. doi: 10.1016/j.clnu.2018.01.012

12. Ni L, Chen Q, Zhu K, Shi J, Shen J, Gong J, et al. The influence of extracorporeal membrane oxygenation therapy on intestinal mucosal barrier in a porcine model for post-traumatic acute respiratory distress syndrome. J Cardiothorac Surg. 2015;10:20. doi: 10.1186/s13019-015-0211-3

13. Zhao L, Luo L, Chen J, Xiao J, Jia W, Xiao Y. Utilization of extracorporeal membrane oxygenation alleviates intestinal ischemia-reperfusion injury in prolonged hemorrhagic shock animal model. Cell Biochem Biophys. 2014;70(3):1733-40. doi: 10.1007/s12013-014-0121-3

14. Wollersheim T, Müller MC, Weber-Carstens S. ECMO patients. En: Berger MM. Critical Care Nutrition Therapy for Non-nutritionists. Suiza: Springer; 2018. p. 29-39. doi: 10.1007/978-3-319-58652-6

15. Wollersheim T, Frank S, Müller MC, Skrypnikov V, Carbon NM, Pickerodt PA, et al. Measuring Energy Expenditure in Extracorporeal Lung Support Patients (MEEP)-Protocol, Feasibility and Pilot Trial. Clin Nutr. 2018;37(1):301-7. doi: 10.1016/j.clnu.2017.01.001

16. De Waele E, van Zwam K, Mattens S, Staessens K, Diltoer $\mathrm{M}$, Honoré $\mathrm{PM}$, et al. Measuring resting energy expenditure during extracorporeal membrane oxygenation: preliminary clinical experience with a proposed theoretical model. Acta Anaesthesiol Scand. 2015;59(10):1296-1302. doi: 10.1111/ aas. 12564

17. Pelekhaty SL, Galvagno Jr SM, Lantry JH, Dolly KN, Herr DL, Kon ZN, et al. Are Current Protein Recommendations for the 
Critically ill Adequate for Patients on VV ECMO: Experience from a High-Volume Center. JPEN J Parenter Enteral Nutr. 2020;44(2):220-6. doi: 10.1002/jpen.1602

18. Bear DE, Smith E, Barrett NA. Nutrition Support in Adult Patients Receiving Extracorporeal Membrane Oxygenation. Nutr Clin Pract. 2018;33(6):738-46. doi: 10.1002/ncp.10211
19. Estensen K, Shekar K, Robins E, McDonald C, Barnett AG, Fraser JF. Macro- and micronutrient disposition in an ex vivo model of extracorporeal membrane oxygenation. Intensive Care Med Exp. 2014;2(1):29. doi: 10.1186/s40635-0140029-7 